\section{Severe Disease Caused by Community-Associated MRSA ST398 Type V, Australia, 2017}

\author{
Geoffrey W. Coombs, Stanley Pang, \\ Denise A. Daley, Yung Thin Lee, \\ Sam Abraham, Marcel Leroi
}

Author affiliations: PathWest Laboratory Medicine WA, Murdoch, Western Australia, Australia (G.W. Coombs); Murdoch University, Murdoch (G.W. Coombs, S. Pang, Y.T. Lee, S. Abraham); Australian Group on Antimicrobial Resistance, Murdoch (D.A. Daley); Austin Health, Heidelberg, Victoria, Australia (M. Leroi)

DOI: https://doi.org/10.3201/eid2501.181136

Using whole-genome sequencing, we identified a community-associated methicillin-resistant Staphylococcus aureus (CA-MRSA) sequence type (ST) 398 type $\mathrm{V}$ (5C2\&5) isolate (typically found in China) in Australia in 2017. This CA-MRSA ST398 variant was highly virulent, similar to other related CA-MRSAs of ST398. This strain should be monitored to prevent more widespread dissemination.

$\mathrm{T}$ he Australian Group on Antimicrobial Resistance (http://agargroup.org.au/) manages multiple national antimicrobial drug resistance surveillance programs, including the Australian Staphylococcus Sepsis Outcome Programme. This program involves 38 hospitals across Australia continuously providing antimicrobial MIC data and demographic data on episodes of Staphylococcus aureus sepsis. Specimens are referred to a central reference laboratory where whole-genome sequencing is performed for all methicillin-resistant $S$. aureus (MRSA) isolates.

In 2017, a MRSA sequence type (ST) 398 type V $(5 \mathrm{C} 2 \& 5)$ isolate, typically referred to as livestock-associated MRSA (LA-MRSA), which has been isolated in many parts of the world including Australia $(1,2)$, was cultured from a 56-year-old man from Singapore who was working as a chef in a suburb of Melbourne, Victoria, Australia. He sought hospital care for a 2-week history of a nonspecific illness and was found to have mitral valve endocarditis with embolic disease involving the spleen, brain, and lungs. Because his MRSA bacteremia failed to resolve after 10 days of vancomycin therapy, he required a mechanical mitral valve replacement. His bacteremia resolved 24 hours after valve replacement. Because of glycopeptide hypersensitivity and concerns of vancomycin heteroresistance, he was prescribed various nonglycopeptide antimicrobial drug therapies (e.g., clindamycin monotherapy, moxifloxacin monotherapy) for variable durations throughout the remainder of his treatment. While he was in the hospital, acute renal injury developed, requiring hemodialysis support. After 3 months' hospitalization, he was transferred to a hospital in Singapore. At the time of transfer, he was improving and undergoing rehabilitation. We were not able to establish if the patient had direct contact with livestock, but as a chef, he presumably had contact with raw meat.

We identified the patient's isolate (S23009-2017) as $S$. aureus by matrix-assisted laser desorption/ionization time-of-flight mass spectrometry using the Bruker MALDI Biotyper (https://www.bruker.com/) and confirmed this finding by DNA microarray using the $S$. aureus Genotyping Kit 2.0 (Alere Technologies, https:// alere-technologies.com/). We performed susceptibility testing by using the VITEK 2 automated microbiology system (bioMérieux, https://www.biomerieux.com/) and performed whole-genome sequencing of the isolate using the Illumina NextSeq 550 Sequencing System (https:// www.illumina.com/). We performed DNA extraction on an overnight subculture using the Invitrogen MagMAX DNA Multi-Sample Kit (ThermoFisher, https://www. thermofisher.com/). We prepared a library of the extracted DNA using the Illumina Nextera XT Library Preparation Kit and sequenced libraries having 150-bp paired-end chemistries. We identified single-nucleotide polymorphisms and performed core genome alignments using Snippy version 3.2 (https://github.com/tseemann/snippy). We constructed a phylogenetic tree using the resulting single-nucleotide polymorphisms in MEGA version 7.0 (https://www.megasoftware.net/) with the maximum parsimony algorithm. We identified antimicrobial resistance and virulence genes, multilocus sequence type, staphylococcal cassette chromosome mec (SCCmec) type, and spa type using available pipelines (https://cge.cbs.dtu.dk/ services/) and confirmed the virulence and antimicrobial resistance gene profile by DNA microarray. We performed a phylogenetic comparison of S23009-2017 with 22 previously published MRSA ST398 whole-genome sequences and rooted the tree with an outgroup of single-locus Panton-Valentine leukocidin (PVL)-positive variants.

We identified S23009-2017 as a PVL-negative, t011carrying, MRSA ST398 type V (5C2\&5) isolate. spa type t011 and SCCmec element type V (5C2\&5) are frequently identified in LA-MRSA ST398 isolates $(3,4)$. However, unlike LA-MRSA ST398, which is typically phenotypically multidrug resistant and harbors multiple antimicrobial drug resistance genes, including tetM (5), S230090-2017 was only resistant to $\beta$-lactams (penicillin and oxacillin) and harbored only the blaZ and mecA antimicrobial drug resistance genes. Furthermore, S23009-2017 harbored the sak, chp, and scn human evasion genes, which are not typically found in LA-MRSA ST398 (6). 

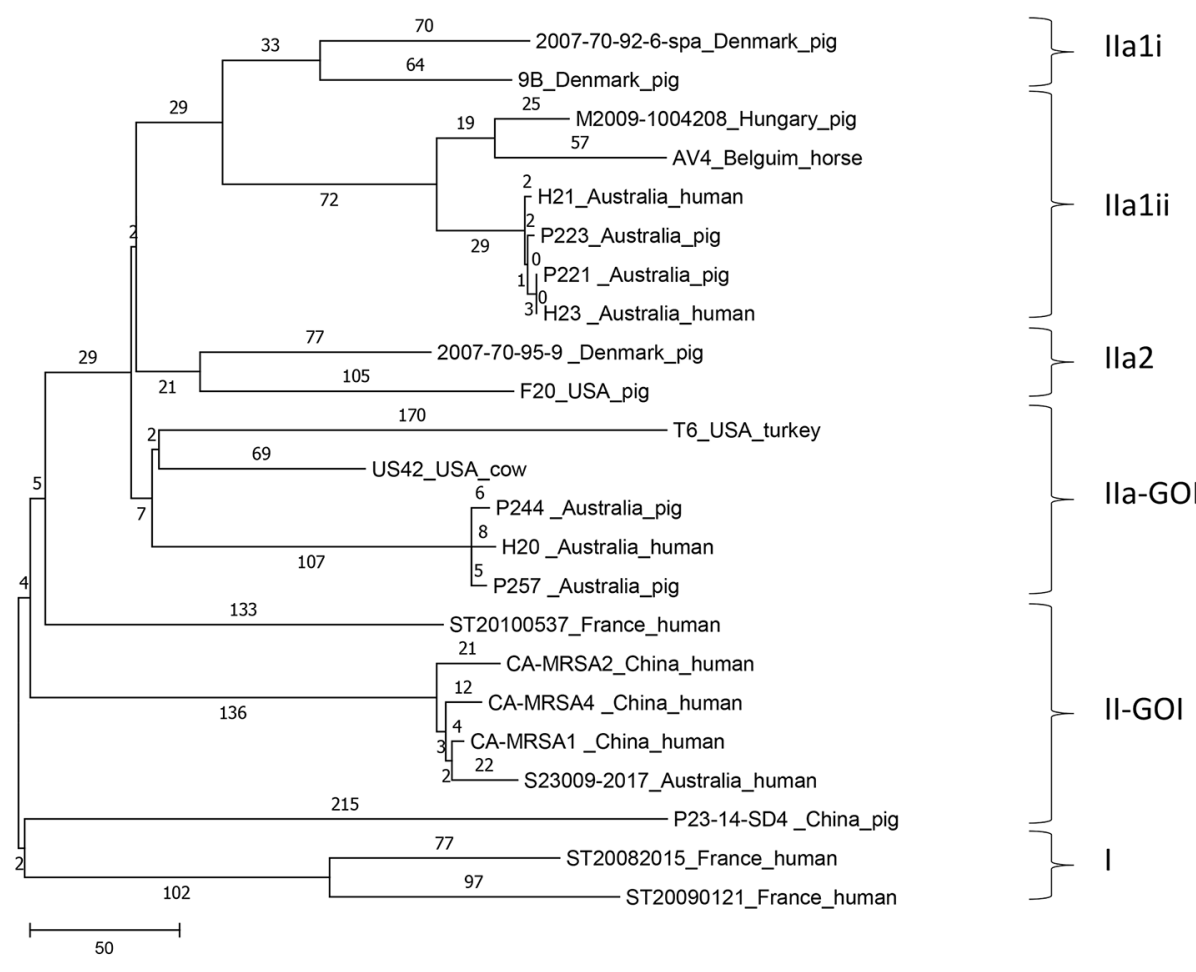

Figure. Phylogenetic tree of MRSA sequence type (ST) 398 isolate S23009-2017, recovered from a man in Australia in 2017, and related MRSA ST398 isolates from around the world $(2,6,7)$. The tree was constructed by using single-nucleotide polymorphism differences and is rooted with MRSA ST398 isolates containing a single Panton-Valentine leukocidin locus. Isolates are grouped into clusters as described by Price et al. (6). Scale bar represents number of nucleotide substitutions per residue. CAMRSA, community-associated MRSA; GOI, gene of interest; MRSA, methicillin-resistant Staphylococcus aureus.

Phylogenetic analysis showed S23009-2007 had a much closer relationship to the PVL-negative MRSA ST398 isolates from China reported by $\mathrm{He}$ et al. than to LA-MRSA ST398 isolates from Australia (Figure) (7). These isolates are grouped within the II-GOI (group of interest) clade from the Price et al. study of worldwide MRSA ST398 isolates (6).

He et al. reported several cases of severe and fatal infections with community-associated MRSA (CA-MRSA) ST398 and proposed that the strain arose from humanadapted predecessors and not from a livestock-adapted strain. Although all of the China isolates harbored a type $\mathrm{V}$ SCCmec, only 1 isolate harbored the type V (5C2\&5) SCCmec element found in S23009-2017. Like S23009-2017, CA-MRSA ST398 isolates from China harbor sak, chp, and scn genes and lack the tetM resistance gene.

MRSA ST398 has not been previously reported to cause serious disease in Australia. Although LA-MRSA ST398 is frequently identified in pig herds in Australia, the isolate from this patient was not LA-MRSA but CA-MRSA and presumably originated in China. Because of immigration irregularities, we were not able to investigate whether the patient had visited China shortly before his illness or if his house companions were from China. Unlike LA-MRSA ST398, CA-MRSA ST398 has been shown to be highly virulent and has become the predominant CA-MRSA circulating in Shanghai, China. Thus, continued monitoring of this strain's epidemiology and preventing its widespread transmission are essential.

\section{Acknowledgments}

The authors thank the staff of the Australian Commission on Safety and Quality in Health Care (https://www.safetyandquality. gov.au/) and the participants of the Australian Group on Antimicrobial Resistance (http://agargroup.org.au/).

This study was partially funded by the Australian Commission on Safety and Quality in Health Care.

\section{About the Author}

Dr. Coombs is Chair of Public Health at Murdoch University, Murdoch, Australia, and Chair of the Australian Group on Antimicrobial Resistance. His research focuses on the antimicrobial drug resistance and molecular epidemiology of S. aureus and Enterococcus faecium.

\section{References}

1. Cuny C, Friedrich A, Kozytska S, Layer F, Nübel U, Ohlsen K, et al. Emergence of methicillin-resistant Staphylococcus aureus (MRSA) in different animal species. Int J Med Microbiol. 2010;300:109-17. http://dx.doi.org/10.1016/j.ijmm.2009.11.002

2. Sahibzada S, Abraham S, Coombs GW, Pang S, Hernández-Jover M, Jordan D, et al. Transmission of highly virulent communityassociated MRSA ST93 and livestock-associated MRSA ST398 between humans and pigs in Australia. Sci Rep. 2017;7:5273. http://dx.doi.org/10.1038/s41598-017-04789-0

3. Stegger M, Lindsay JA, Sørum M, Gould KA, Skov R. Genetic diversity in CC398 methicillin-resistant Staphylococcus aureus isolates of different geographical origin. Clin Microbiol Infect. 2010;16:1017-9. http://dx.doi.org/10.1111/j.1469-0691. 2009.03003.x 
4. Monecke S, Slickers P, Gawlik D, Müller E, Reissig A, Ruppelt-Lorz A, et al. Variability of SCCmec elements in livestockassociated CC398 MRSA. Vet Microbiol. 2018;217:36-46. http://dx.doi.org/10.1016/j.vetmic.2018.02.024

5. Kadlec K, Fessler AT, Hauschild T, Schwarz S. Novel and uncommon antimicrobial resistance genes in livestock-associated methicillin-resistant Staphylococcus aureus. Clin Microbiol Infect. 2012;18:745-55. http://dx.doi.org/10.1111/j.1469-0691. 2012.03842.x

6. Price LB, Stegger M, Hasman H, Aziz M, Larsen J, Andersen PS, et al. Staphylococcus aureus CC398: host adaptation and emergence of methicillin resistance in livestock. MBio. 2012; 3:e00305-11. http://dx.doi.org/10.1128/mBio.00305-11

7. He L, Zheng HX, Wang Y, Le KY, Liu Q, Shang J, et al. Detection and analysis of methicillin-resistant human-adapted sequence type 398 allows insight into community-associated methicillin-resistant Staphylococcus aureus evolution. Genome Med. 2018;10:5. http://dx.doi.org/10.1186/s13073-018-0514-9

Address for correspondence: Geoffrey W. Coombs, Murdoch University, Antimicrobial Resistance and Infectious Diseases Laboratory, School of Veterinary and Life Sciences, Murdoch, WA, Australia; email: g.coombs@murdoch.edu.au

\section{Candida auris Sternal Osteomyelitis in a Man from Kenya Visiting Australia, 2015}

\section{Christopher H. Heath, ${ }^{1}$ John R. Dyer, Stanley Pang, Geoffrey W. Coombs, Dianne J. Gardam ${ }^{1}$}

\footnotetext{
Author affiliations: Royal Perth Hospital, Perth, Western Australia, Australia (C.H. Heath); University of Western Australia, Crawley, Western Australia, Australia (C.H. Heath); Fiona Stanley Hospital, Murdoch, Western Australia, Australia (C.H. Heath, J.R. Dyer); PathWest Laboratory Medicine WA, Murdoch (C.H. Heath, S. Pang, G.W. Coombs, D.J. Gardam); Murdoch University, Murdoch (S. Pang, G.W. Coombs)
}

DOI: https://doi.org/10.3201/eid2501.181321

In Australia in 2015, Candida auris sternal osteomyelitis was diagnosed in a 65-year-old man with a history of intensive care treatment in Kenya in 2012 and without a history of cardiac surgery. The isolate was South Africa clade III. Clinicians should note that $C$. auris can cause low-grade disease years after colonization.
C andida auris, first reported in Japan in 2009 (1), is an emerging pathogen that has caused severe disease in hospitalized patients in many countries, including India, South Africa, Spain, the United Kingdom, the United States, and Venezuela (2-4). In July 2015, a 65 -year-old man from Kenya visiting Australia for the first time sought treatment in Perth, Western Australia, Australia, for chronically discharging sternal sinus persisting for $>1$ year. His active medical problems included severe hypercapneic chronic obstructive pulmonary disease with pulmonary hypertension, ischemic heart disease, and chronic kidney impairment. In July 2012, he had unstable angina treated by coronary stenting that was complicated by cardiac arrest with cardiopulmonary resuscitation, which resulted in sternal injuries and a 3 -month intensive care unit hospitalization in Nairobi, Kenya. At hospital admission, computed tomography scan of the chest showed a $3.3-\mathrm{cm}$ subcutaneous collection and bony changes from chronic sternal osteomyelitis (Figure). Surgical debridement confirmed sternal osteomyelitis with parasternal abscesses. Posaconazole was given as pragmatic oral therapy, and trough serum levels of $2.0 \mathrm{mg} / \mathrm{L}$ at week 2 and $2.60 \mathrm{mg} / \mathrm{L}$ at week 4 were achieved. The patient died from progressive cardiorespiratory failure 3 months later.

Deep operative sternal bone samples yielded a yeast on Difco CHROMagar Candida medium (Becton Dickinson, https://www.bd.com/) that did not produce pseudohyphae or germ tubes. The isolate grew well at $40^{\circ} \mathrm{C}$ and $42^{\circ} \mathrm{C}$ but not $45^{\circ} \mathrm{C}$. Matrix-assisted laser desorption/ionization timeof-flight mass spectrometry (MALDI version 3.1; Bruker Daltonics, https://www.bruker.com/) identified the pathogen as Candida auris (score $>2.1$ ).

Sequencing of the $18 \mathrm{~S}$ rDNA internal transcribed region and 28S rDNA D1-D2 regions confirmed pathogen identification (Appendix Figure 1, https://wwwnc. cdc.gov/EID/article/25/1/18-1321-App1.pdf). We edited the DNA sequences, assembled consensus sequences using SeqScape (Applied Biosystems, https://www.thermofisher.com/us/en/home/brands/applied-biosystems.html), and performed sequence alignments with BLAST (https:// blast.ncbi.nlm.nih.gov/Blast.cgi). The internal transcribed regions of our isolate matched $100 \%$ with $C$. auris reference strain KP131674.1. The D1-D2 regions of the isolate also matched $100 \%$ with those of multiple $C$. auris isolates (GenBank accession nos. JQ219331-2, KM000828, KM000830, KU321688). Susceptibility testing with the Sensititre YeastOne YO10 panel (Trek Diagnostic Systems, https://www.thermofisher.com/) showed fluconazole resistance (MIC $>256 \mathrm{mg} / \mathrm{L}$ ) and posaconazole susceptibility (MIC $0.06 \mathrm{mg} / \mathrm{L}$ ) (Appendix Table).

\footnotetext{
${ }^{1}$ These authors contributed equally to this article.
} 